\title{
Statistical acceleration of interstellar pick-up ions in co-rotating interaction regions
}

\author{
N. A. Schwadron, L. A. Fisk \\ Department of Atmospheric, Oceanic, and Space Sciences, University of Michigan
}

\section{G. Gloeckler}

Department of Physics and ISTP, University of Maryland

and, Department of Atmospheric, Oceanic, and Space Sciences, University of Michigan

\begin{abstract}
Observations from the SWICS instrument on Ulysses reveal that there is little evidence for the acceleration of interstellar pick-up ions at the forward and reverse shocks which surround Co-rotating Interactions Regions (CIR's) in the solar wind. Rather, the pick-up ions exhibit strong acceleration in regions within the CIR where there are increased variations in the magnitude of the magnetic field. A simple model is presented in which the pick-up ions are statistically accelerated by transit-time damping the magnitude variations. The model yields the observed distribution functions for the accelerated pick-up ions.
\end{abstract}

\section{Introduction}

It has been well established that particles with energies of order $1 \mathrm{MeV}$ per nucleon can be accelerated at the forward and reverse shocks which surround Co-rotating Interaction Regions (CIR's), where high and low speed streams interact in the solar wind [e.g. Barnes and Simpson 1976; McDonald et al. 1976; Fisk and Lee 1980]. Although recent theoretical investigations [Zank et al. 1996; Lee et al. 1996] have put forth shock acceleration mechanisms for lower energy particles, the appropriate acceleration mechanism has not yet been clearly established. In a recent paper, Gloeckler et al. [1994] discussed a specific CIR event in which there were detailed observations of the behavior of interstellar pick-up ions. These particles enter the heliosphere as interstellar neutral particles and are then ionized and picked-up by the solar wind, acquiring energies $\sim 1 \mathrm{keV}$ per nucleon. In the Gloeckler et al. [1994] event, the pick-up ions show little evidence of being accelerated at the surrounding shocks, but rather exhibit a pronounced high energy tail at energies greater than $1 \mathrm{keV}$ per nucleon, in the region interior to the CIR, where there is strong turbulence in the solar wind.

Copyright 1996 by the American Geophysical Union.

Paper number 96GL02833.

0094-8534/96/96GL-02833\$05.00
It is the purpose of this paper to extend the study of Gloeckler et al. [1994] to encompass many different CIR events by analyzing data obtained with the Solar Wind Ion Composition Spectrometer (SWICS) on Ulysses [Gloeckler et al., 1992]. The measurements reported here were made between Febuary 19, 1992, and July 18, 1992, while Ulysses remained near the solar equatorial plane, at about 5.5 AU from the Sun and encountered 22 forward and reverse shocks surrounding CIR's. The presence of these CIR's resulted in substantial acceleration of pick-up protons.

\section{Observations}

Plotted in Figure 1 as diamonds is the average pick-up proton distribution observed during the 150 day interval. When interstellar ions are first picked up by the solar wind, they acquire a thermal speed equal to the solar wind flow speed, in the frame moving with the solar wind. Thus, in the spacecraft frame they can have speeds in the range $0 \leq v_{\mathrm{S} / \mathrm{C}} \leq 2 u$, where $v_{\mathrm{S} / \mathrm{C}}$ is the particle speed in the spacecraft frame and $u$ is the solar wind speed. In quiet times, then, there is a sharp cutoff in the pick-up ion distribution at speeds of $v_{\mathrm{S} / \mathrm{C}}=\mathbf{2 u}$. Particles with speeds $v_{\mathrm{S} / \mathrm{C}}>2 u$ result from local acceleration in disturbed conditions.

A useful measure of the extent to which the interstellar ions experience acceleration is the quantity:

$$
\bar{E}=\frac{m}{3 n_{r}} \int_{2 u}^{6 u} d^{3} v_{\mathrm{S} / \mathrm{C}} v_{\mathrm{S} / \mathrm{C}}^{2} f_{\mathrm{S} / \mathrm{C}}
$$

where $n_{r}=\int_{1.8 u}^{2 u} d^{3} v_{\mathrm{S} / \mathrm{C}} f_{\mathrm{S} / \mathrm{C}} ; f_{\mathrm{S} / \mathrm{C}}$ is the distribution function of pick-up ions as seen in the spacecraft frame; and $m$ is particle mass. The integral in (1) will yield the energy density of the pick-up ions in the accelerated tail of the distribution, at $v_{\mathrm{S} / \mathrm{C}} \geq 2 u$. The effectiveness of the acceleration will depend on the number of particles that are available to be accelerated, which as discussed by Fisk et al. [1996] can vary by a factor of $\sim 2$. Hence we normalize the energy density by dividing it by a reference density $n_{r}$ in the speed range $1.8 u \leq v_{\mathrm{S} / \mathrm{C}} \leq$ $2 u$, which is a measure of the particles available for acceleration. Thus, $\bar{E}$ is the mean energy imparted by the acceleration per available pick-up ion. 


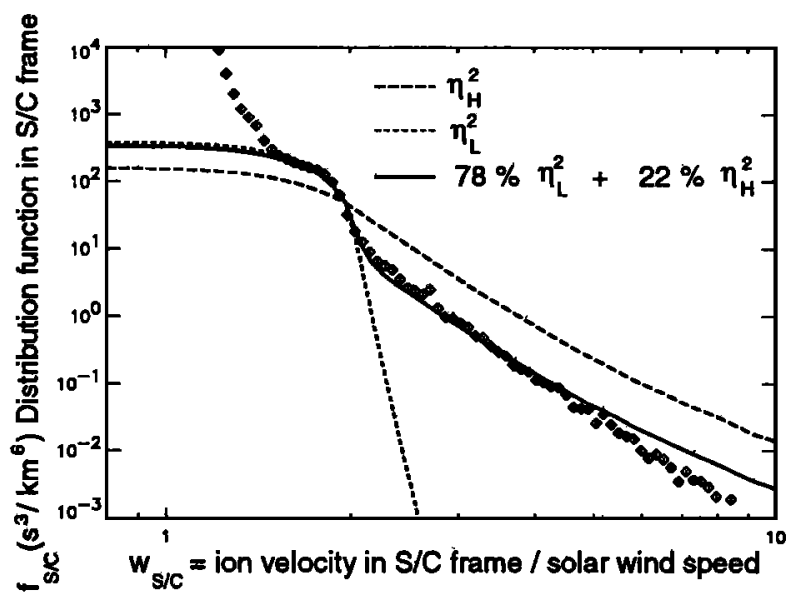

Figure 1. Plotted as diamonds is the average pickup proton distribution function observed between day 50 and 200 of 1992 in the spacecraft reference frame. Model results, as described in section 3, are plotted as the solid curves.

We have used 5-day running averages and determined $\bar{E}$ for interstellar pick-up hydrogen during the 150 days from February 19 to July 18, 1992; the results are plotted in Figure 2. Also shown with vertical lines are the locations of the forward and reverse shocks during this interval [Balogh et al. 1994].

We have also used the Ulysses Common Data Pool Tape (which provides data in 256 second intervals) to determine the variations in the magnitude of the magnetic field. We use 1-day averages to determine the magnitude of the mean field $B_{0}$ and then average the square of the variations in the field magnitude,

$$
\eta^{2}=\left\langle\left(B-B_{0}\right)^{2} / B_{0}^{2}\right\rangle
$$

over a 5-day period. The results are also plotted in Figure 2.

The 5-day averages used in Figure 2 were necessary to yield a comparatively smooth function of time for $\eta^{2}$. However, to compare the effects of shocks with the

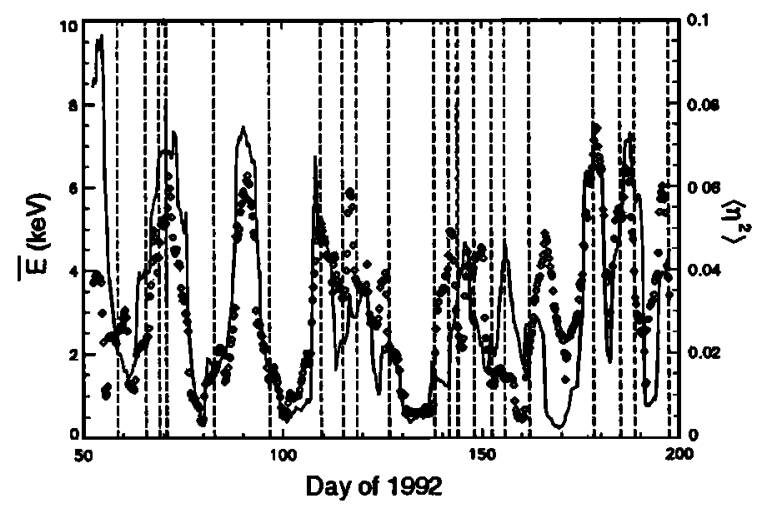

Figure 2. The mean energy per available pick-up ion defined in (1), plotted as the solid curve, and $\left\langle\eta^{2}\right\rangle$ defined in (2), plotted as diamonds, vs. day of 1992. Vertical dashed lines represent the passage of shocks. effects of magnitude fluctuations, we have used smaller 1-day averages. Each data point in this time series has an associated 1 day-averaged value for $\bar{E}$ and $\eta^{2}$. We group this data into 8 bins, each centered on a value of $\bar{E}=\bar{E}_{i}$ (for $i=1 \ldots 8$ ) such that a data point falls into a bin if its associated value for $\bar{E}$ falls in the range $\bar{E}_{i}-\Delta \bar{E} / 2<\bar{E} \leq \bar{E}_{i}+\Delta \bar{E} / 2$, with $\Delta \bar{E}=1 \mathrm{keV}$. We then form averages for $\bar{E}$ and $\eta^{2}$ from the data contained in each bin. The averages are displayed in Figure 3. We have also calculated the percentage of data in each bin which fall within one day of a shock; this also is displayed in Figure 3. It can be seen that less than $10 \%$ of the data can be explained by shock acceleration and that there is a positive correlation between the presence of magnitude fluctuations and acceleration.

Magnitude variations in the magnetic field can be strongly damped by, e.g. transit-time damping [e.g. Fisk 1976]. In fact, with the exception of regions such as CIR's, which with their interacting streams can generate local turbulence, the amplitudes of the magnitude fluctuations in the solar wind tend to be very low, i.e. the ambient fluctuations are mainly Alfvenic [e.g. Smith 1995]. A logical conclusion is that the acceleration of pick-up ions is not due to shocks, but rather is due to statistical acceleration through the damping of magnitude variations in the magnetic field.

\section{Theory}

Consider a simple model for the statistical acceleration of the pick-up hydrogen. We shift into the frame co-rotating with the Sun, where the particle distribution function should be stationary in time. We assume, since Ulysses is in the the equatorial plane and the levels of turbulence are high, that spatial diffusion is not significant; the particles experience sufficient pitch-angle scattering so that the primary transport mechanism is convection with the solar wind. In this frame the solar wind velocity is along the mean magnetic field direction. Because we are considering very low speed particles, of order the solar wind speed $u$, we measure the particle

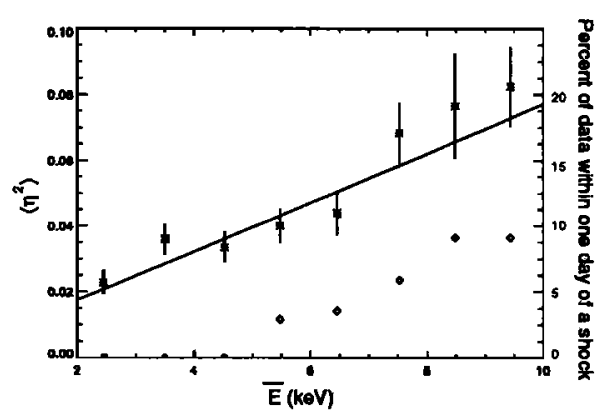

Figure 3. Plotted as stars are averages for $\bar{E}$, defined in (1), and averages for $\eta^{2}$ defined in (2), formed by binning the data according to $\bar{E}$. Plotted as diamonds are the percentage of data within each $\bar{E}$ bin which fall within one day of a shock. The solid line represents the results of our analytic model as described in section 3 . 
speeds $v$ in the frame moving with the solar wind. The behavior of the isotropic particle distribution function $f_{0}$ is then governed by [e.g., Fisk, 1976]:

$$
\begin{array}{r}
u \frac{\partial f_{0}}{\partial r}-\frac{2 u}{3 r} v \frac{\partial f_{0}}{\partial v}-\frac{1}{v^{2}} \frac{\partial}{\partial v}\left(v^{2} D_{v v} \frac{\partial f_{0}}{\partial v}\right)= \\
\beta_{p}\left(\frac{r_{0}}{r}\right)^{2} \frac{n_{H}(r, \theta)}{4 \pi u^{2}} \delta(v-u) .
\end{array}
$$

The first term describes convection with the solar wind ( $u$ is the radial flow speed of the solar wind); the second term describes adiabatic deceleration; the third term represents the statistical acceleration - diffusion in velocity space with a diffusion coefficient $D_{v v}$; the term on the right side is a source term from the continuous ionization of interstellar hydrogen. The production rate, $\beta_{p}$, for interstellar pick-up hydrogen is referenced to $1 \mathrm{AU} ; n_{H}(r, \theta)$ is the density of interstellar neutral hydrogen at a radial distance $r$ from the Sun, and for an angle $\theta$ between the solar wind direction and the upstream direction of the neutrals. For the interstellar neutral hydrogen density we have used Thomas' [1978] cold neutral model, with a loss rate of $6 \cdot 10^{-7} \mathrm{~s}^{-1}$ referenced to $1 \mathrm{AU}$; a value of 0.65 for the ratio of the radiation pressure to the gravitational force; a neutral bulk velocity of $20 \mathrm{~km} / \mathrm{s}$; and a neutral hydrogen density of $0.08 \mathrm{~cm}^{-3}$ in the interstellar medium [Gloeckler et al. 1993].

We consider the case in which statistical acceleration results from the transit time damping of magnitude fluctuations in the interplanetary magnetic field [Fisk 1976]. We have calculated the coefficient of velocity diffusion, $D_{v v}$, using quasi-linear theory [Fisk 1976] with a power spectrum that falls off as $k^{-3 / 2}$ at large wavenumber $k$ and resembles observed power-spectra [Jokipii and Coleman 1968; Sari and Ness 1969]; with a magnetosonic wave speed of $50 \mathrm{~km} / \mathrm{sec}$; with a mean magnetic field of $B_{0}=0.76 \mathrm{nT}$ as obtained from the magnetic field data; and with a representative pitchangle cosine $\mu=0.3$. We also assume that the turbulence in CIR's is quite elongated, i.e. $\lambda_{\|} \gg \lambda_{1}$, where $\lambda_{\|}$is the correlation length parallel to the mean magnetic field and $\lambda_{\perp}$ is the perpendicular correlation length. The normal expansion of the solar wind should stretch field variations in the azimuthal as opposed to the radial direction, and CIR's have a generally elongated geometry. In Figure 4, we plot $1 / \eta^{2} \cdot D_{v v} / v^{2}$ vs. $w$ with a correlation length perpendicular to the field $\lambda_{\perp}=0.01 \mathrm{AU} ;$ a correlation length parallel to the field $\lambda_{\|}=30 \cdot \lambda_{\perp}$; and a solar wind speed $470 \mathrm{~km} / \mathrm{sec}$. As can be seen for this case of elongated turbulence, the velocity diffusion coefficient is well approximated by

$$
\frac{D_{v v}}{v^{2}}=\eta^{2} D_{0} w
$$

in the velocity range $v \mu<v_{w} \lambda_{\|} / \lambda_{\perp}$, where $w=v / u$; for these values of $\lambda_{\perp}$ and $\lambda_{\|}, D_{0} \approx 4 \cdot 10^{-6} \mathrm{~s}^{-1}$.

With the simple form of $D_{v v}$ given in (4) and assuming $\eta^{2}$ is constant, (3) can be solved analytically:

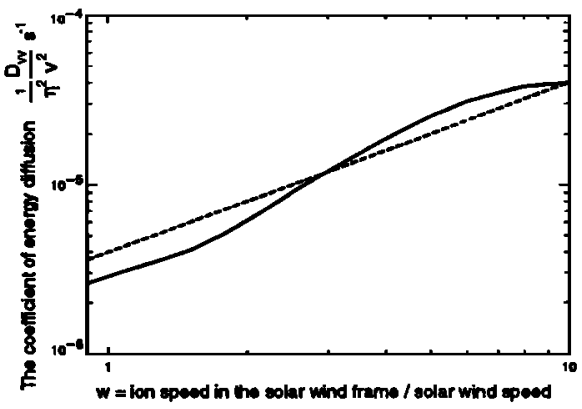

Figure 4. The velocity diffusion coefficient, $1 / \eta^{2}$. $D_{v v} / v^{2}$ vs. normalized velocity $w=v / u$ in the solar wind frame. The solid curve is the quasi-linear prediction for the parameters listed in section 3 [Fisk 1976] and the dashed line is the approximation given in (4).

$$
\begin{aligned}
f_{0}(r, w, \theta)= & \frac{1}{8 \pi u^{3}} \frac{r_{0} \beta_{x}}{u} \frac{r_{0}}{r} w^{-2} \int_{0}^{1} d x x^{-(4 / 3) \frac{n_{H}(r x, \theta)}{\Delta(r, x)}} \\
\cdot & \exp \left(-\left(w^{-1}+x^{-2 / 3}\right) /(2 \Delta(r, x))\right) \\
& \cdot \mathrm{I}_{4}\left(w^{-1 / 2} x^{-1 / 3} / \Delta(r, x)\right),
\end{aligned}
$$

where $\Delta(r, x)=3 \eta^{2} D_{0} r\left(1-x^{1 / 3}\right) /(2 u)$, and $I_{4}(x)$ is a modified Bessel function of order 4 .

Consider, then, detailed fits to the observed spectra. For $\eta^{2}$, we find from the 1-day averaged magnetic field data that there is a range of values, from a high value of $\eta_{H}^{2} \sim 0.12$ to a low value of $\eta_{L}^{2} \sim 0.005$. We consider the case in which the observed distribution functions are the result of a mixture of these high and low values of $\eta^{2}$, i.e. Ulysses crosses flux tubes with different values of $\eta^{2}$. We use the value of $D_{0}=4 \cdot 10^{-6} \mathrm{~s}^{-1}$ consistent with the dashed line in Figure 4 , and we adjust the production rate $\beta_{p}$, within allowable limits, to give the correct normalization of the predicted distributions.

Shown in Figure 1 is the predicted distribution function compared to the average distribution function, observed over 150 days. The distribution is calculated in the frame of the solar wind through (5) and integrated over the instrument response function of SWICS to yield the predicted curve in Figure 1. The mixture of $\eta^{2}$ is $78 \%$ low fluctuations $\left(\eta_{L}^{2}\right)$ and $22 \%$ high fluctuations $\left(\eta_{H}^{2}\right)$, consistent with the mean value of $\eta^{2}=0.03$ over the 150 day period; with a production rate $\beta_{p}=3.5 \cdot 10^{-7} \mathrm{~s}^{-1}$. Note that this mixture of $\eta^{2}$ yields the "knee" in the observed distribution at $v_{\mathrm{S} / \mathrm{C}}=2 u$. There is some disagreement between modeled and observed spectra for $w>5$. This may indicate that there is an energy threshold for statistical acceleration, above which shocks may play an important role for acceleration.

Based on this analytic theory, $\bar{E}$ is calculated as a function $\eta^{2}$ and is plotted in Figure 3. The agreement between data and theory is excellent, yielding a reduced chi squared, $\tilde{\chi}^{2}=0.99$ with an expected value of 1.0 .

In summary the observed spectra can be reproduced using statistical acceleration by transit time damping assuming that the turbulence geometry is elongated parallel to the magnetic field, and assuming flux tubes 
with varying levels of turbulence contribute to the observed spectra.

\section{Concluding Remarks}

We have presented observational evidence that interstellar pick-up hydrogen is not accelerated at the forward and reverse shocks which surround CIR's, but rather is accelerated interior to the CIR, where there are relatively strong magnitude variations in the magnetic field. We have also presented a simple transport model which statistically accelerates pick-up ions by the transit-time damping of magnitude fluctuations. Assuming the turbulence geometry is elongated parallel to the mean magnetic field, and assuming flux tubes with varying levels of turbulence contribute to the observed spectra, the modeled distribution functions are consistent with observed spectra.

These results suggest strongly that there is a threshold for shock acceleration. The forward and reverse shocks of CIR's are quasi-perpendicular; the magnetic field tends to be aligned with the shock front. Thus, particles must need a sufficient speed, so that following their first interaction with the shock front they can propagate upstream to return for further interactions. Presumably this threshold speed is above the speeds of the pick-up ions. For particles that originate at low energies, acceleration in CIR's must be a two step process. The particles first undergo statistical acceleration by interacting with the magnitude fluctuations. They then reach speeds above the shock acceleration threshold such that they are mobile in the solar wind and can be accelerated at the forward and reverse shocks which surround the CIR.

Finally we note that these results have implications for the anomalous cosmic ray component, which originates as pick-up ions [Fisk et al. 1974]. It is believed that pick-up ions undergo substantial acceleration and achieve anomalous component energies at the termination shock of the solar wind [Pesses et al. 1981]. Presumably the termination shock is even more perpendicular than CIR forward and reverse shocks in the inner heliosphere. Hence the pick-up ions cannot be injected directly into acceleration at the termination shock, but rather they must achieve some pre-acceleration as they are convected outward with the solar wind.

Acknowledgments. This work was supported by NASA/JPL contract 955460 . N. Schwadron was supported by NASA grant NST-51323.

\section{References}

Balogh, A., et al., Interplanetary shock waves: Ulysses observations in an out of ecliptic plane, ESLAB Symposium, April 1994.

Barnes, C. W., and J. A. Simpson, Evidence for interplanetary acceleration of nucleons in corotating interaction regions, Astrophys. J., 210, L91,1976.

Fisk, L. A., B. Kozlovsky, and R. Ramaty, An interpretation of the observed oxygen and nitrogen enhancements in lowenergy cosmic rays, Astrophys. J., 185, L105, 1974.

Fisk, L. A., The acceleration of energetic particles in the interplanetary medium by transit time damping, J. Geophys. Res., 81, 4633, 1976.

Fisk, L. A., and M. A. Lee, Shock acceleration of energetic particles in corotating interaction regions in the solar wind, Astrophys. J., 237, 620, 1980.

Fisk, L. A., N. A. Schwadron, and G. Gloeckler, Implications of fluctuations in the distribution functions of interstellar pick-up ions for the scattering of low rigidity particles, Geophys. Res. Lett., in press, 1996.

Gloeckler, G., et al., The solar wind ion composition spectrometer, Astron. Astrohpys. Suppl. Ser., 92, 267, 1992.

Gloeckler, G., et al., Detection of interstellar pickup hydrogen in the solar system, Science, 261, 70, 1993.

Gloeckler, G., et al., Acceleration of interstellar pickup ions in the disturbed solar wind observed on Ulysses, J. Geophys. Res., 99, 17637, 1994.

Jokipii, J. R., and P. J. Coleman, Jr., The cosmic ray diffusion tensor and its variation observed with Mariner 4, J. Geophys. Res., 73, 5495, 1968.

Lee, M. A., V. D. Shapiro, and R. Z. Sagdeev, Pickup ion energization by shock surfing, J. Geophys. Res., 101, 4777, 1996.

McDonald, F. B., B. J. Tegarden, J. H. Trainor, and T. T. von Rosenvinge, The interplanetary acceleration of energetic nucleons, Astrophys. J., 203, L149, 1976.

Pesses, M. E., J. R. Jokipii, and D. Eichler, Cosmic ray drift, shock acceleration, and the anomalous component of cosmic rays, Astrophys. J., 246, L85, 1981.

Sari, J. W., and N. F. Ness, Power spectral studies of the interplanetary magnetic field, Solar Phys., 8, 155, 1969.

Smith, et al., Ulysses observations of latitude gradients in the heliospheric magnetic field: radial component and variances, Space Science Reviews, 72, 165, 1995.

Thomas, G. E., The interstellar wind and its influence on the interplanetary environment, Ann. Rev. Earth Planet. Sci., 6, 173, 1978.

Zank, G. P., H. L. Pauls, I. H. Cairns, and G. M. Webb, Interstellar pickup ions and quasi-perpendicular shocks: Implications for the termination shock and interplanetary shocks, J. Geophys. Res., 101, 457, 1996.

N. A. Schwadron and L. A Fisk, University of Michigan, Dept. of AOSS, Space Research Bldg, Ann Arbor MI 48109-2143

(Received May 6, 1996; revised July 19, 1996; accepted August 29, 1996.) 